\title{
Genetic Association Studies: Discovery of the Genetic Basis of Renal Disease
}

\author{
Marion Verduijn ${ }^{\mathrm{a}}$ Kitty J. Jager ${ }^{\mathrm{b}}$ Carmine Zoccali ${ }^{\mathrm{c}}$ Friedo W. Dekker ${ }^{\mathrm{a}}$ \\ a Department of Clinical Epidemiology, Leiden University Medical Center, Leiden, and bepartment of Medical \\ Informatics, ERA-EDTA Registry, Academic Medical Center, University of Amsterdam, Amsterdam, The Netherlands; \\ ${ }^{C}$ CNR-IBIM Clinical Epidemiology and Pathophysiology of Renal Diseases and Hypertension, Renal and \\ Transplantation Unit, Ospedali Riuniti, Reggio Calabria, Italy
}

Key Words

Epidemiology $\cdot$ Genetics $\cdot$ Nephrology

\begin{abstract}
Genetic association studies are a means to investigate the causal role of genes in diseases in order to unravel pathways involved in the etiology of disease. There are two types of genetic association studies: hypothesis-driven studies, i.e. candidate gene studies, targeting genes with a known or presumed role in pathways or diseases of interest, and nonhypothesis-driven studies, i.e. genome-wide association studies, aiming for the discovery of new genetic associations. This educational article is an introduction to genetic association studies for nephrologists and researchers in the domain of kidney disease.

Copyright $\odot 2011$ S. Karger AG, Basel
\end{abstract}

\section{Introduction}

With the success of the Human Genome Project that identified up to 25,000 genes and sequenced the 3.3 billion base pairs that make up human DNA, the interest in genetic studies is greatly enhanced. Numerous studies have been performed examining associations between genetic variants and diseases with the aim to unravel pathways involved in the etiology of diseases. With evidence from genetic association studies of genes being associated with disease, the corresponding gene products represent potential disease markers and are potential targets for therapeutic interventions.

The genetic component of diseases and the number of genes involved differ substantially among diseases. Renal disorders like polycystic kidney disease or Alport syndrome are examples of monogenetic (or Mendelian) diseases with a single gene being involved. In contrast, diabetic nephropathy is an example of a so-called complex, multifactorial disease: multiple genes are involved as well as multiple environmental factors. Up to now, the causative genes underlying a variety of Mendelian renal disorders have been successfully identified while the discovery of the genetic basis of complex renal diseases turned out to be much more difficult [1]. This forms an important challenge for future research.

This educational article is an introduction to genetic association studies for nephrologists and researchers in the domain of kidney disease. All concepts presented in italics are briefly defined in a glossary.

\section{KARGER \\ Fax +4161306 1234 E-Mail karger@karger.ch} www.karger.com

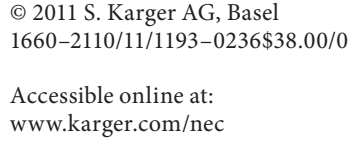

Marion Verduijn

Department of Clinical Epidemiology, Leiden University Medical Center

PO Box 9600

NL-2300 RC Leiden (The Netherlands)

Tel. +31 71526 4394, E-Mail m.verduijn@lumc.nl 


\section{Human Variation}

Although two unrelated persons share more than 99\% of the human genome, the number of potential variations between two persons is still high given that the human DNA is made up of more than 3 billion base pairs [2]. Genetic variants that occur with a frequency of less than $1 \%$ in populations are known as mutations. Polymorphisms are genetic variants that occur in populations with frequencies of at least $1 \%$, and are as such potential determinants in genetic association studies. Polymorphisms may take different forms, including single-nucleotide polymorphisms (SNPs), the presence or absence of a stretch of DNA (insertions/deletions), and repeating patterns of DNA. The unique rs-number enables the identification of a polymorphism in databases such as dbSNP [3] for detailed information on its location in the human genome and the prevalence in different ethnic groups.

\section{Candidate Gene versus Genome-Wide Association Studies}

Two types of genetic association studies can be distinguished: hypothesis-driven studies, i.e. candidate gene studies, and non-hypothesis-driven studies, i.e. genomewide association studies. In candidate gene studies, genes with a known or presumed role in pathways or diseases are targeted, given the results of previous studies. An example of a candidate gene study is a study on the association between the ApoE polymorphism and estimated glomerular filtration rate in 5,583 individuals from the Third National Health and Nutrition Examination Survey (NHANES III) [4]; the authors motivated this study by referring to previous studies showing that the ability of ApoE to bind and clear lipids in the kidney is directly related to structural instability and repair of the glomerular structure of the kidney.

Alternatively, in genome-wide association studies, most or all genes are screened in order to examine how much of the genetic variability associates with a disease. In a recent genome-wide association study in 23,812 participants of European descent, some new variants associated with serum creatinine levels were identified [5]. Genome-wide association studies provide as such useful information for further investigation in candidate gene studies. For more recent examples of (and current findings in) candidate gene studies and genome-wide association studies in chronic kidney disease, we refer to a recent review by O'Seaghdha and Fox [6].
Table 1. Calculation of HWE for the Thr256Ser polymorphism (rs4918) in 1,043 patients starting on dialysis, with allele A being the Thr allele, and allele a being the Ser allele

\begin{tabular}{lllll}
\hline Genotypes & $\begin{array}{l}\text { Observed } \\
\text { numbers }\end{array}$ & $\begin{array}{l}\text { Observed } \\
\text { frequency }\end{array}$ & $\begin{array}{l}\text { Expected } \\
\text { frequency }\end{array}$ & $\begin{array}{l}\text { Expected } \\
\text { number }\end{array}$ \\
\hline Homozygote AA & 472 & 0.453 & 0.459 & 478.6 \\
Heterozygote Aa & 469 & 0.450 & 0.437 & 455.9 \\
Homozygote aa & 102 & 0.098 & 0.104 & 108.6 \\
Allele A & & 0.677 & & \\
Allele a & & 0.323 & \multicolumn{2}{c}{$\chi^{2}=0.86 ; \mathrm{p}=0.35$} \\
\end{tabular}

The observed frequency of allele $\mathrm{A}$ is the observed frequency of genotype AA + half of the observed frequency of genotype Aa; the observed frequency of allele a is the observed frequency of genotype aa + half of the observed frequency of genotype Aa.

The expected frequency of genotype AA is the observed frequency of allele $\mathrm{A} \times$ the observed frequency of allele $\mathrm{A}$; the expected frequency of genotype Aa is twice the observed frequency of allele $\mathrm{A} \times$ the observed frequency of allele a; the expected frequency of genotype aa is the observed frequency of allele $\mathrm{a} x$ the observed frequency of allele a.

Given the expected frequencies and the size of the population, the expected number of patients can be calculated per genotype. Differences between the observed and expected number of genotypes are subsequently tested using a $\chi^{2}$ test.

\section{Genotyping Errors and Hardy-Weinberg Equilibrium}

In genetic association studies, one or multiple genetic variants, or combinations of variants (known as haplotypes) are the determinant of interest. Therefore, unbiased and accurate genotyping of the variants is an essential requisite for valid results. Genotyping error may occur due to problems with the biological material (e.g. old or low-quality samples) or with the genotyping technique. Inconsistency of genotype frequencies with the Hardy-Weinberg equilibrium (HWE) might indicate the presence of genotyping errors [7].

Genotype frequencies are consistent with HWE when the observed frequencies do not deviate from the expected frequencies given constant allele and genotype frequencies in the population. Table 1 shows an example of HWE calculation in a study on the association between a polymorphism in the alpha-2-Heremans-Schmid glycoprotein gene and mortality in 1,043 patients starting on dialysis [8].

Genetic variants can deviate from HWE for other reasons than genotyping errors. HWE assumes random

Nephron Clin Pract 2011;119:c236-c239 
mating in the population, which does not hold for inbreeding and migration, and when selection according to genotype is present. In a study on genetic variants in a diseased population, such as patients with chronic kidney disease, selection is present when the variant is associated with that disease, resulting in a deviation from HWE. In case-control studies, HWE of a genetic variant is therefore commonly tested in the control (nondiseased) group only.

\section{Causality and Confounding}

Genetic variants in genetic association studies are analyzed in the same manner as nongenetic determinants in conventional epidemiology. When an association is observed between a genetic variant and a disease in a given population, this could indicate that the genetic variant has a causal role. However, it could also be that the variant has no causal role, rather a neighboring variant (i.e. a variant that is in linkage disequilibrium with the genetic variant of interest), thereby confounding the observed association. Moreover, the observed association can be due to population stratification. This occurs when the allele frequencies of the genetic variant and the distribution of the disease vary substantially between different (ethnic) subgroups in the study population; for instance, both end-stage renal disease and the GG genotype of a polymorphism in the CNR1 gene, which plays a role in alcohol dependence, are highly present in blacks compared to other subgroups. In this case, a spurious association is induced between the genetic variant and the disease at the population level.

Apart from confounding by nearby genetic variants and ethnicity, confounding by phenotypic or environmental factors is no common issue in genetic association studies. According to Mendel's second law (Gregor Mendel, 1822-1884), an individual's genotype is randomly assigned from his/her parental genotypes at gamete formation, as such eliminating regular confounding [9]. When factors are associated with a genetic variant, they are generally in the causal pathway between the variant and the disease. This is an important reason not to adjust for these factors in order to estimate the causal effect of the genetic variant.

This can be illustrated by a candidate gene study on the role of the endothelin-1 (EDN1) gene in renal impairment in a nondiabetic population-based cohort of 7,291 participants [10]. The EDN1 gene was believed to play an important role in blood pressure regulation. All partici- pants were genotyped for two SNPs in the EDN1 gene; both SNPs were combined in haplotypes. In the crude analysis, a $30 \%$ increased risk of microalbuminuria was suggested for participants who were homozygous for the GN haplotype [1.3, 95\% confidence interval (CI): 0.9-2.0]; the risk was 0.9 (95\% CI: 0.4-1.8) after adjustment for factors including hypertension. The adjusted risk is not an estimate of the causal association between the EDN1 gene and microalbuminuria: the effect of the gene was 'taken away' by adjustment, which indicates that factors adjusted for are potentially in the causal pathway.

\section{False-Positive and False-Negative Findings}

Given the large number of variants being evaluated in genome-wide association studies, all having a small prior probability of being associated with the disease of interest, these studies carry a substantial risk of false-positive findings. Adjustment for multiple comparisons by Bonferroni correction, however, is regarded as overly conservative, and other methods have been suggested, including the false-discovery rate [11]. As the prior probability of promising variants is still relatively low in candidate gene studies and these studies often have limited sample size, they have a risk of false-positive findings too. Evidence of the presence of an association between a genetic variant and a disease is greatly enlarged when it has been identified in different independent populations. Replication studies are conducted for this purpose.

In complex diseases, small effect sizes of variants are being expected. Sufficiently large study populations are required in order to avoid negative findings of not detecting existing effects. This holds for genome-wide association studies and candidate gene studies. The genotype frequency of the genetic variant of interest is an additional determinant of sample size.

In order to increase sample sizes in renal studies, data harmonization and pooling has become increasingly important. New international initiatives on building collaborative infrastructures have appeared in renal research in order to facilitate data pooling and replication studies [12].

\section{Conclusions}

Unraveling the basis of complex renal diseases is an ongoing challenge in nephrology. Genetic association studies are a means to identify new candidate genes and 
to estimate their causal effect. This article provided an introduction to genetic association studies. For more details, we refer the reader to useful educational series that have been published in The Lancet $[13,14]$ and JAMA [2, $11,15]$. Guiding principles for reporting results of genetic association studies as proposed in the STrengthening the Reporting of Genetic Assocation studies (STREGA) Statement [16] are recommended to be used in order to increase the transparency of genetic association studies in nephrology.

\section{Acknowledgement}

This work was supported by the applied GENomic stratEgies for Treatment and Prevention of Cardiovascular death in Uraemia and End stage REnal disease (GENECURE) project (www. genecure.eu), a Specific Targeted Research or Innovation Project, funded by the European Commission under the Sixth Framework Programme as FP6-037696.

\section{Disclosure Statement}

The results presented in this paper have not been published previously in whole or part.

\section{Glossary}

Alleles: variant forms of a gene at a locus; a single allele is inherited from each parent.

Gene: a stretch of DNA which encodes for a particular protein.

Genetic variants: variation in genes, including SNPs, insertions and deletions, and repeating patterns of DNA.

Genotype: genetic make-up of an individual with regard to genetic variants.

Haplotypes: combinations of SNPs; usually, a limited number of haplotypes can be used to cover most of the genetic variation within a population due to linkage.

Hardy-Weinberg equilibrium: a situation in which the genotype frequencies of a population are constant from generation to generation.

Mutation: a genetic variant that occurs infrequently in the population (i.e. less than $1 \%$ ).

Linkage: tendency of loci to be coinherited due to close physical proximity.

Linkage disequilibrium: a measure of linkage indicating whether a nonrandom association between two alleles at different loci is present.

Locus: physical location of a gene or other genetic marker.

Polymorphism: a genetic variant that occurs frequently in the population (i.e. more than $1 \%$ ).

Population stratification: a form of confounding by ethnicity due to differences in allele frequencies in subgroups in a population.

Single-nucleotide polymorphism: a polymorphism in which one specific nucleotide in the DNA is altered.

\section{References}

1 McKnight AJ, Currie D, Maxwell AP: Unravelling the genetic basis of renal disease; from single gene to multifactorial disorders. J Pathol 2010;220:198-216.

2 Attia J, Ioannidis JPA, Thakkinstian A, McEvoy M, Scott RJ, Minelli C, Thompson J, Infante-Rivard C, Guyatt G: How to use an article about genetic association. A. Background concepts. JAMA 2009;301:74-81.

3 dbSNP, available at www.ncbi.nlm.nih.gov/ snp

-4 Chu AY, Parekh RS, Astor BC, Coresh J, Berthier-Schaad Y, Smith MW, Shuldiner AR, Kao WHL: Association of APOE polymorphism with chronic kidney disease in a nationally representative sample: a Third National Health and Nutrition Examination Survey (NHANES III) Genetic Study. BMC Med Genet 2009;10:108.

$\checkmark 5$ Chambers JC, Zhang W, Lord GM, et al: Genetic loci influencing kidney function and chronic kidney disease. Nat Genet 2010;42: 373-375.

-6 O'Seaghdha CM, Fox CS: Genetics of chronic kidney disease. Nephron Clin Pract 2011; 188:c55-c63.
7 Hosking L, Lumsden S, Lewis K, Yeo A, McCarthy L, Bansal A, Riley J, Purvis I, Xu CF: Detection of genotyping errors by HardyWeinberg equilibrium testing. Eur J Hum Genet 2004;12:395-399.

8 Verduijn M, Prein RA, Stenvinkel P, Carrero JJ, le Cessie S, Witasp A, Nordfors L, Krediet RT, Boeschoten EW, Dekker FW: Is fetuin-A a mortality risk factor in dialysis patients or a mere risk marker? A Mendelian randomization approach. Nephrol Dial Transplant 2011;26:239-245.

-9 Verduijn M, Siegerink B, Jager KJ, Zoccali C Dekker FW: Mendelian randomization: use of genetics to enable causal inference in observational studies. Nephrol Dial Transplant 2010;25:1394-1398.

10 Pinto-Sietsma SJ, Herrmann SM, SchmidtPetersen K, Niu T, Hillege HL, Janssen WM, de Zeeuw D, de Jong P, Kreutz R: Role of the endothelin-1 gene locus for renal impairment in the general nondiabetic population. J Am Soc Nephrol 2003;14:2596-2602.
11 Attia J, Ioannidis JPA, Thakkinstian A, McEvoy M, Scott RJ, Minelli C, Thompson J, Infante-Rivard C, Guyatt G: How to use an article about genetic association. B. Are the results of the study valid? JAMA 2009;301: 191-197.

12 Brenchley PE, Lindholm B, Dekker FW, Navis G: Translating knowledge of the human genome into clinical practice in nephrology dialysis and transplantation: the Renal Genome Network (ReGeNet). Nephrol Dial Transplant 2006;21:2681-2683.

13 Burton PR, Tobin MD, Hopper JL: Key concepts in genetic epidemiology. Lancet 2005; 366:941-951.

14 Cordell HJ, Clayton DG: Genetic association studies. Lancet 2005;366:1121-1131.

15 Attia J, Ioannidis JPA, Thakkinstian A, McEvoy M, Scott RJ, Minelli C, Thompson J, Infante-Rivard C, Guyatt G: How to use an article about genetic association. C. What are the results and will they help me in caring for my patients? JAMA 2009;301:304-308.

16 Little J, Higgins JPT, Ioannidis JPA, et al: STrengthening the REporting of Genetic Association studies (STREGA): an extension of the STROBE statement. Ann Intern Med 2009;150:206-215. 